\title{
Alcalinidade total normalizada na Zona Econômica Exclusiva da região Norte (Brasil)
}

\author{
Normalized total alkalinity in the Exclusive Economic Zone of the North Region (Brazil)
}

\author{
M. L. S. Santos ${ }^{1 *} ;$ A. O. Bordalo ${ }^{2}$, A. C. Silva $^{3} ;$ M. Araújo ${ }^{3}$ \\ ${ }^{1}$ Instituto Socioambiental e dos Recursos Hídricos/Laboratório de Química Ambiental, Universidade Federal Rural da \\ Amazônia, CEP 66077-530, Belém-Pará,Brasil \\ ${ }^{2}$ Centro de Ciências Naturais e Tecnologia/Laboratório de Hidrocarbonetos, Universidade do Estado do Pará, CEP \\ 66095-100, Belém-Pará,Brasil \\ ${ }^{3}$ Departamento de Oceanografia/Laboratório de Oceanografia Física Estuarina e Costeira,Universidade Federal de \\ Pernambuco, CEP 50739-540, Recife-Pernambuco, Brasil \\ *mdelssantos@yahoo.com.br \\ (Recebido em 15 de março de 2016; aceito em 20 de julho de 2016)
}

\begin{abstract}
No ambiente marinho uma equação inversa entre a alcalinidade total e a salinidade, denominada de alcalinidade total normalizada (ATN) tem como finalidade mostrar uma relação linear entre estes dois parâmetros. O objetivo deste trabalho foi avaliar a distribuição superficial e vertical da ATN ao longo da Zona Econômica Exclusiva-ZEE da região Norte do Brasil, com os dados obtidos nas Operações Norte III e Norte IV, dentro do âmbito do Programa de Avaliação do Potencial Sustentável de Recursos Vivos na Zona Econômica Exclusiva (REVIZEE). Os dados demonstraram que o sistema do dióxido de carbono ao longo da ZEE Norte mantém o $\mathrm{pH}$ dentro da faixa esperada para o ambiente marinho. Os menores valores de $\mathrm{pH}$ (7,48 Norte III e 7,38 Norte IV), de AT $\left(1971 \mu \mathrm{mol} \mathrm{kg}{ }^{-1}\right.$, Norte III e $1878 \mu \mathrm{mol} \mathrm{kg}{ }^{-1}$ Norte IV) e de ATN (2295 $\mu \mathrm{mol} \mathrm{kg} \mathrm{k}^{-1}$ Norte III, $2293 \mu \mathrm{mol} \mathrm{kg} \mathrm{k}^{-1}$ Norte IV) na camada superficial, foram observados na área com influência da descarga do rio Amazonas, ou seja, com baixos valores de salinidade (28,31 Norte III e 24,00 Norte IV). As massas de águas na coluna de água, encontradas na região, tiveram diferenças na concentração de ATN. Essas diferenças podem ser associadas aos processos de degradação da matéria orgânica, e a formação ou dissolução do carbonato de cálcio.
\end{abstract}

Palavras-chave: REVIZEE, salinidade, massas de água.

An inverse equation between the total alkalinity and the salinity, denominated of normalized total alkalinity (NTA) to show a linear relationship among those parameters. The objective of this work was to evaluate the superficial and vertical distribution of NTA along the Economic Exclusive Zone-EEZ of the North Brazil, with the data obtained in the Operations North III and North IV, inside of the Program of Evaluation of Sustainable Potential of Living Resources in the Exclusive Economic Zone (REVIZEE). The data demonstrated that the system of the carbon dioxide maintains the $\mathrm{pH}$ inside of the expected strip for the seawater. In the superficial layer, the smallest $\mathrm{pH}$ values (7,48 North III and 7,38 North IV), NTA (1971 $\mu$ mol $\mathrm{kg}^{-1}$, North III and $1878 \mu \mathrm{mol} \mathrm{kg}{ }^{-1}$ North IV) and of NTA $\left(2295 \mu \mathrm{mol} \mathrm{kg}{ }^{-1}\right.$ North III, $2293 \mu \mathrm{mol} \mathrm{kg}^{-1} \mathrm{North}$ IV), were observed in the area with influence of the discharge of the Amazon river, with low salinity values $(28,31$ North III and 24,00 North IV). The waters masses found in the water column in this area, had differences in the concentration of NTA. Those differences can be associated to the processes of organic matter degradation, and the formation or dissolution of the calcium carbonate.

Keywords: REVIZEE, salinity, waters masses.

\section{INTRODUÇÃO}

Aproximadamente $40 \%$ da fonte antrópica de $\mathrm{CO}_{2}$ liberada para atmosfera foi retida nos oceanos [1]. A entrada de $\mathrm{CO}_{2}$ ocasiona aumento da concentração de íons $\mathrm{H}^{+}$na água e consequentemente torna o meio ácido. Contudo, o sistema dióxido de carbono no ambiente marinho funciona como tampão e mantém o pH em um limite estreito [2]. Este equilíbrio do $\mathrm{pH}$ é relacionado à presença do par ácido-base conjugado fraco, como por exemplo, os ânions $\mathrm{HCO}_{3}^{-}$e $\mathrm{CO}_{3}{ }^{2-}$. 
$\mathrm{O}$ índice para representar a concentração de todos os ânions básicos do sistema de $\mathrm{CO}_{2}$ dos oceanos é a alcalinidade total (AT). Esse parâmetro é uma importante propriedade físico-química da água do mar e tem um papel fundamental nos vários processos químicos e biológicos. Segundo Burke e Atkinson (1988) [3] destacam-se: (a) os $\mathrm{HCO}_{3}{ }^{-}$e $\mathrm{CO}_{3}{ }^{2-}$ maiores ânions de ácidos fracos na água do mar, o que permite observar o comportamento conservativo com a salinidade dos oceanos; (b) a precipitação do carbonato de cálcio diminui a AT, e como resultado AT pode ser indicativo da calcificação de processos biogeoquímicos que envolvem espécies de $\mathrm{CO}_{2} ; \mathrm{e}$ (c) a fotossíntese e a respiração das comunidades biológicas que modificam a concentração de carbono inorgânico dissolvido.

No ambiente marinho uma equação inversa entre a alcalinidade total e a salinidade, denominada de alcalinidade total normalizada foi empregada por Millero (1996) [4] com a finalidade de mostrar uma relação linear entre estes dois parâmetros.

Na Zona Econômica Exclusiva (ZEE) da região Norte do Brasil, destaca-se a presença das águas oriundas do rio Amazonas na área da plataforma continental. O rio Amazonas é responsável pelo estabelecimento da maior zona estuarina do mundo. Aproximadamente $16 \%$ da água doce e $10 \%$ dos sedimentos lançados anualmente nas águas do Oceano Attântico são oriundos desse rio [6].

$\mathrm{O}$ rio Amazonas é considerado como um dos maiores rios do mundo, em termos de descarga de água (100.000- $\left.220.000 \mathrm{~m}^{3} \mathrm{~s}^{-1}\right)$ e de sedimentos (11- $13 \times 10^{8}$ t.ano $\left.^{-1}\right)$ [7], com um fluxo de componentes dissolvido de $2-3 \times 10^{8}$ tons.ano ${ }^{-1}$ [8], e uma variabilidade intra-anual, que ocorre sazonalmente com mínimo $\left(0,9 \times 10^{5}\right.$ $\left.\mathrm{m}^{3} \mathrm{~s}^{-1}\right)$ de outubro a dezembro e máximo $\left(2,3 \times 10^{5} \mathrm{~m}^{3} \mathrm{~s}^{-1}\right)$ de maio a junho [9].

Trabalhos desenvolvidos na Zona Econômica exclusiva (ZEE) da região Norte do Brasil [10, 11, 12, 13, 14] descrevem uma estabilidade térmica na camada superficial, uma diminuição da temperatura com o aumento da profundidade e a presença da termoclina na área oceânica.

Devido ao compromisso assumido pelo Brasil, quando da ratificação da Convenção das Nações Unidas sobre o Direito do Mar (CNUDM), que atribui aos países costeiros direitos e responsabilidades quanto à exploração, conservação e gestão dos recursos vivos de sua Zona Econômica Exclusiva (ZEE) e à dinâmica interna e evolução da atividade pesqueira nacional, foi criado o Programa "Avaliação do Potencial Sustentável de Recursos Vivos na Zona Econômica Exclusiva" - REVIZEE. Este Programa teve como objetivo central proceder ao levantamento dos potenciais sustentáveis de captura dos recursos vivos na ZEE, que compreende uma faixa que se estende das 12 às 200 milhas marítimas, a partir das linhas de base que servem para medir a largura do mar territorial.

O Programa REVIZEE na Plataforma Continental Norte do Brasil abrangeu a área entre o Cabo Orange/AP e a Foz do rio Parnaíba/PI, e realizou campanhas oceanográficas denominadas: Operação Norte I (1995), Norte II (1997), Norte III (1999) e Norte IV (2001), a bordo do Navio Oceanográfico “ANTARES" da Marinha do Brasil.

Os objetivos deste trabalho foram: (i) avaliar a distribuição superficial e na coluna de água da alcalinidade total (AT) e da alcalinidade total normalizada (ATN) ao longo da ZEE Norte, com os dados obtidos nas Operações Norte III e Norte IV; (ii) relacionar os dados de AT e ATN com os valores de temperatura, salinidade e $\mathrm{pH}$.

\section{MATERIAL E MÉTODOS}

As estações oceanográficas executadas durante as Operações Norte III e Norte IV (Figura 1), a bordo do Navio Oceanográfico "ANTARES", foram efetuadas com a finalidade de obter informações de natureza biótica e abiótica na ZEE Norte, entre o Cabo Orange/AP e a Foz do rio Parnaíba/PI, dentro do âmbito do Programa de Avaliação do Potencial Sustentável de Recursos Vivos na Zona Econômica Exclusiva (REVIZEE).

As coletas hidrológicas foram realizadas com garrafas de Niskin, acopladas ao conjunto Rosette/CTD. As profundidades das amostragens foram: superfície, $50 \%$ e $1 \%$ de penetração de luz, início, meio e fim da termoclina.

Em ambas as operações foram obtidos e analisados a bordo: (i) salinidade e temperatura registrados por um CTD (Conductivity, Temperature, Depth) SeaBird SBE 9-plus ligado à unidade de bordo SBE 11 acoplado a uma Rosette; (ii) $\mathrm{pH}$ medido com um pHmetro da marca Hanna; (iii) alcalinidade total medida pelo método do $\mathrm{pH}$ descrito por Wagener e Carreira (1997).

Para obtenção da alcalinidade total normalizada foi realizado o cálculo: $A T N=A T x 35 / S$, onde, ATN é a alcalinidade total normalizada $\left(\mu \mathrm{mol} \mathrm{kg}{ }^{-1}\right)$, AT alcalinidade total $\left(\mu \mathrm{mol} \mathrm{kg}{ }^{-1}\right), 35$ é o valor da salinidade constante [4].

Os dados de temperatura, salinidade, $\mathrm{pH}$, alcalinidade total, alcalinidade total normalizada, obtidos durante as Operações Norte III e Norte IV, foram analisados através de métodos de estatística descritiva e análise de componentes principais. 


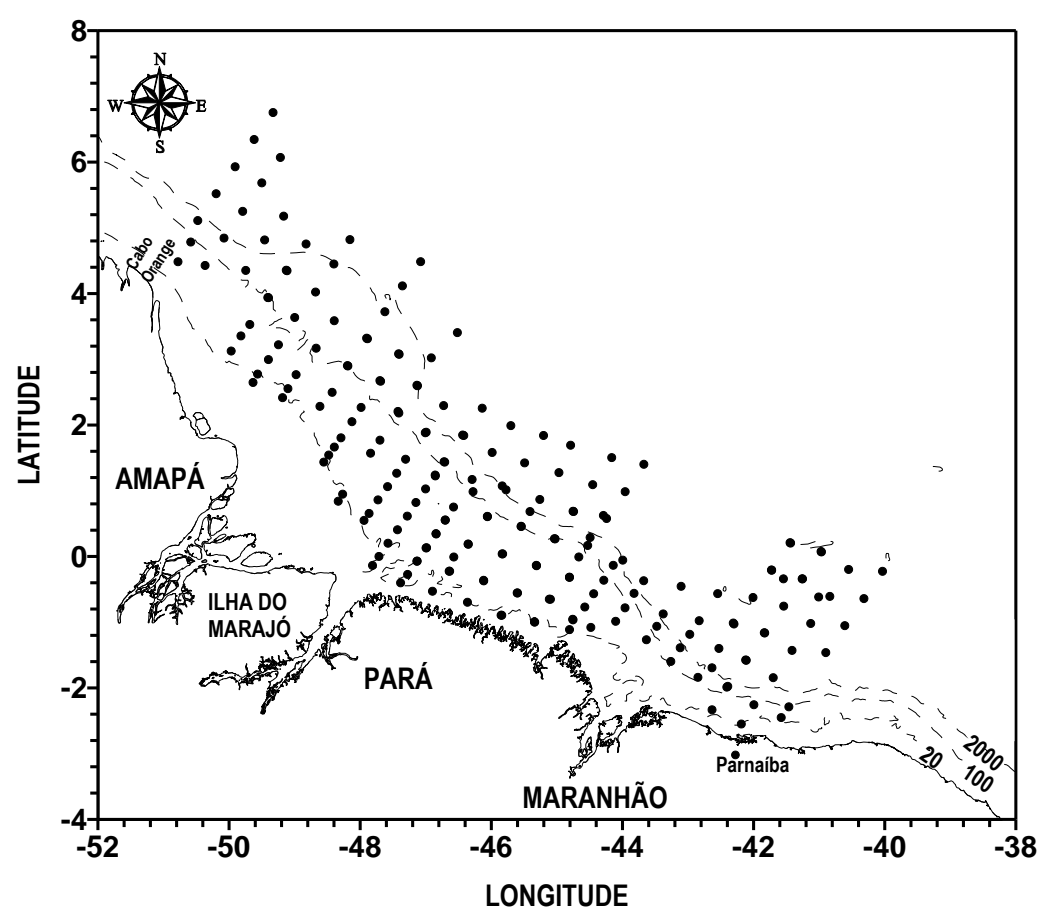

Figura 1: Mapa de localização da área de estudo, os pontos indicam as estações oceanográficas realizadas durante a Operação Norte III e Norte IV/Programa REVIZEE.

\section{RESULTADOS}

No presente trabalho as análises de alcalinidade total foram limitadas as estações com faixas de salinidade superficial de 28,31 a 36,27 (média de 35,06 — 1,76), durante a Operação Norte III; e de 24,00 a 36,42 (média

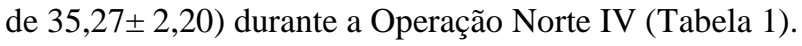

A distribuição vertical da salinidade oscilou de 28,31 a 37,10 (média de 35,42 \pm 8,60), durante a Operação Norte III; e de 24,00 a 36,91 (média de 35,78 \pm 1,26) durante a Operação Norte IV (Tabela 2).

Tabela 1: Estatística descritiva (número de amostras $\left(N^{\circ}\right)$, mínimo, máximo, média) dos valores superficiais (temperatura, salinidade, $\mathrm{pH}$, alcalinidade total - AT, alcalinidade total normalizada - ATN) obtidos nas Operações Norte III e Norte IV, na ZEE Norte.

\begin{tabular}{|c|c|c|c|c|c|}
\hline \multirow{6}{*}{ Operação Norte III } & & $\mathrm{N}^{\mathrm{o}}$ & Mínimo & Máximo & Média \\
\hline & Temperatura $\left({ }^{\circ} \mathrm{C}\right)$ & 91 & 26,82 & 29,25 & $27,93 \pm 0,53$ \\
\hline & Salinidade & 91 & 28,31 & 36,27 & $35,06 \pm 1,76$ \\
\hline & $\mathrm{pH}$ & 92 & 7,48 & 8,34 & $8,16 \pm 0,17$ \\
\hline & $\mathrm{AT}\left(\mu \mathrm{mol} \mathrm{kg} \mathrm{kg}^{-1}\right)$ & 91 & 1971 & 2379 & $2317 \pm 90,40$ \\
\hline & ATN $\left(\mu \mathrm{mol} \mathrm{kg}{ }^{-1}\right)$ & 91 & 2295 & 2436 & $2314 \pm 29,50$ \\
\hline \multirow{5}{*}{ Operação Norte IV } & Temperatura $\left({ }^{\circ} \mathrm{C}\right)$ & 148 & 26,17 & 29,30 & $27,62 \pm 0,74$ \\
\hline & Salinidade & 140 & 24,00 & 36,42 & $35,27 \pm 2,20$ \\
\hline & $\mathrm{pH}$ & 144 & 7,38 & 8,23 & $7,75 \pm 0,13$ \\
\hline & $\mathrm{AT}\left(\mu \mathrm{mol} \mathrm{kg}{ }^{-1}\right)$ & 120 & 1878 & 2386 & $2347 \pm 80,80$ \\
\hline & $\operatorname{ATN}\left(\mu \mathrm{mol} \mathrm{kg}{ }^{-1}\right)$ & 120 & 2293 & 2480 & $2305 \pm 26,70$ \\
\hline
\end{tabular}


Tabela 2: Estatística descritiva (número de amostras $\left(N^{\circ}\right)$, mínimo, máximo, média e desvio padrão) dos valores obtidos na coluna de água (temperatura, salinidade, $\mathrm{pH}$, alcalinidade total - AT, alcalinidade total normalizada - ATN) durante as Operações Norte III e Norte IV, na ZEE Norte.

\begin{tabular}{|c|c|c|c|c|c|}
\hline & & $\mathrm{N}^{\mathrm{o}}$ & Mínimo & Máximo & Média \\
\hline \multirow{5}{*}{ Operação Norte III } & Temperatura $\left({ }^{\circ} \mathrm{C}\right)$ & 559 & 3,01 & 29,25 & $21,49 \pm 8,60$ \\
\hline & Salinidade & 559 & 28,31 & 37,10 & $35,42 \pm 1,14$ \\
\hline & $\mathrm{Ph}$ & 560 & 7,10 & 8,38 & $8,05 \pm 0,24$ \\
\hline & $\mathrm{AT}\left(\mu \mathrm{mol} \mathrm{kg} \mathrm{kg}^{-1}\right)$ & 558 & 1971 & 2421 & $2335 \pm 59,70$ \\
\hline & $\operatorname{ATN}\left(\mu \mathrm{mol} \mathrm{kg}{ }^{-1}\right)$ & 558 & 2284 & 2436 & $2308 \pm 18,10$ \\
\hline \multirow{5}{*}{ Operação Norte IV } & Temperatura $\left({ }^{\circ} \mathrm{C}\right)$ & 633 & 3,68 & 29,3 & $25,02 \pm 5,91$ \\
\hline & Salinidade & 622 & 24,00 & 36,91 & $35,78 \pm 1,26$ \\
\hline & $\mathrm{Ph}$ & 625 & 7,10 & 8,27 & $7,70 \pm 0,17$ \\
\hline & $\mathrm{AT}\left(\mu \mathrm{mol} \mathrm{kg} \mathrm{kg}^{-1}\right)$ & 466 & 1878 & 2409 & $2363 \pm 51,64$ \\
\hline & $\operatorname{ATN}\left(\mu \mathrm{mol} \mathrm{kg}{ }^{-1}\right)$ & 466 & 2287 & 2480 & $2300 \pm 16,55$ \\
\hline
\end{tabular}

Em relação aos dados de temperatura na camada superficial estes oscilaram de $26,82^{\circ} \mathrm{C}$ a 29,25 ${ }^{\circ} \mathrm{C}$ (média de $27,93{ }^{\circ} \mathrm{C} \pm 0,53$ ) durante a Operação Norte III e de $26,17^{\circ} \mathrm{C}$ a $29,30{ }^{\circ} \mathrm{C}$ (média de $\left.27,62^{\circ} \mathrm{C} \pm 0,74\right)$ durante a Operação Norte IV. A distribuição vertical deste parâmetro oscilou de $3,01^{\circ} \mathrm{C}$ a $29,25^{\circ} \mathrm{C}$ (média de $21,49^{\circ} \mathrm{C} \pm 8,60$ ) durante a Operação Norte III e de $3,68^{\circ} \mathrm{C}$ a $29,30^{\circ} \mathrm{C}$ (média de $25,02{ }^{\circ} \mathrm{C} \pm 5,91$ ) durante a Operação Norte IV (Tabelas 1 e 2).

As massas de água na região da Zona Econômica Exclusiva brasileira, na área da Plataforma Continental do Amazonas e área oceânica adjacente são: Água Costeira com temperatura entre $26,00^{\circ} \mathrm{C}$ e $28,87^{\circ} \mathrm{C}$, e salinidade $\leq 34,00$; Água Tropical com temperatura $>18,00{ }^{\circ} \mathrm{C}$ e salinidade > 36,00; Água Central do Atlântico Sul com temperatura entre $5,95^{\circ} \mathrm{C}$ e $18,35^{\circ} \mathrm{C}$, e salinidade entre 34,52 e 36,40; e Água Intermediária da Antártica com temperatura entre $4,92^{\circ} \mathrm{C}$ e $5,90^{\circ} \mathrm{C}$, e salinidade entre 34,48 e 34,78 [16].

No presente trabalho, os valores de salinidade e de temperatura na camada superficial indicam a presença das massas de Água Costeira e Água Tropical. Enquanto, na coluna de água foram observadas a Água Central do Atlântico Sul e Âgua Intermediária da Antártica.

As distribuições dos dados de ATN versus profundidade durante as Operações Norte III e Norte IV foram similares (Figuras 3a e 3b), com os maiores valores na massa de Água Costeira. Na massa de Água Tropical foi observada uma diminuição dos valores de ATN, em ambas as operações.

ATN

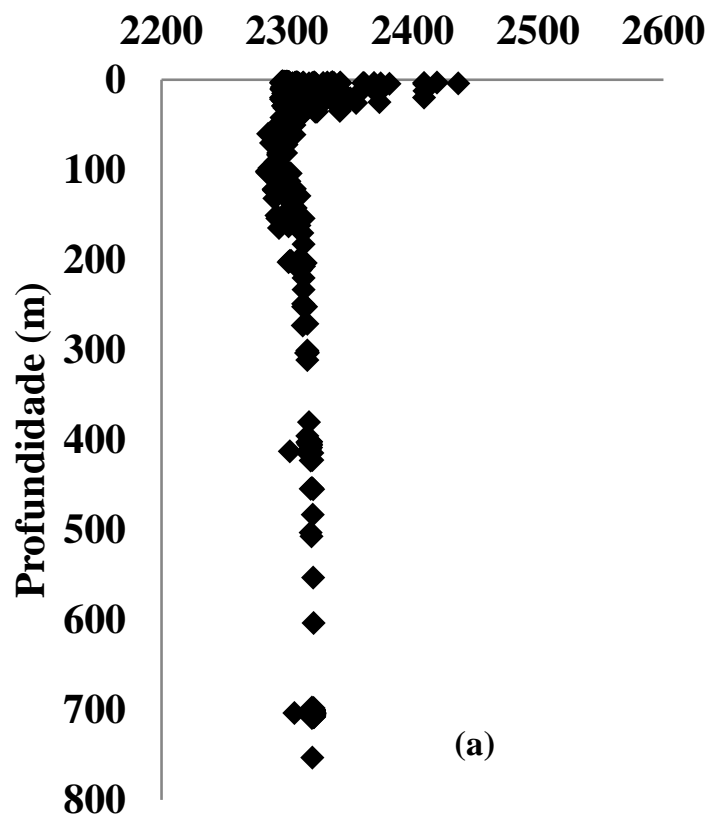

\section{ATN}

22002300240025002600

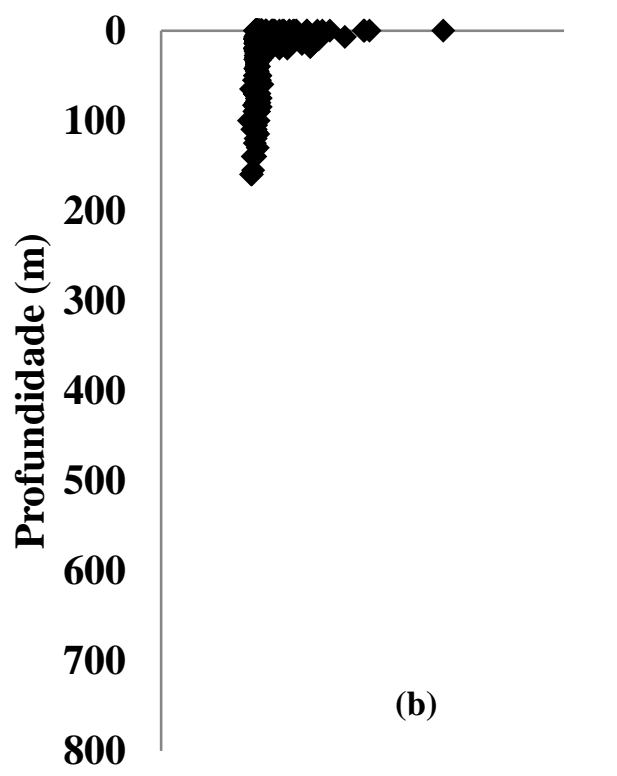


Figura 3: Distribuição vertical da ATN ( $\left.\mu \mathrm{mol} \mathrm{kg}^{-1}\right)$ versus profundidade com dados obtidos durante a Operação Norte III (a) e Norte IV (b).

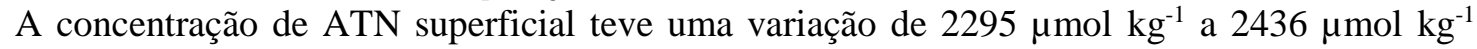
(média de $2314 \mu \mathrm{mol} \mathrm{kg}{ }^{-1} \pm 29,50$ ) durante a Operação Norte III; e de $2293 \mu \mathrm{mol} \mathrm{kg} \mathrm{kg}^{-1}$ a $2480 \mu \mathrm{mol}$ $\mathrm{kg}^{-1}$ (média de $2305 \mu \mathrm{mol} \mathrm{kg}{ }^{-1} \pm 26,70$ ) durante a Operação Norte IV. Em ambas as operações foram observadas o comportamento linear da ATN com a salinidade (Figuras 4a e 4b).

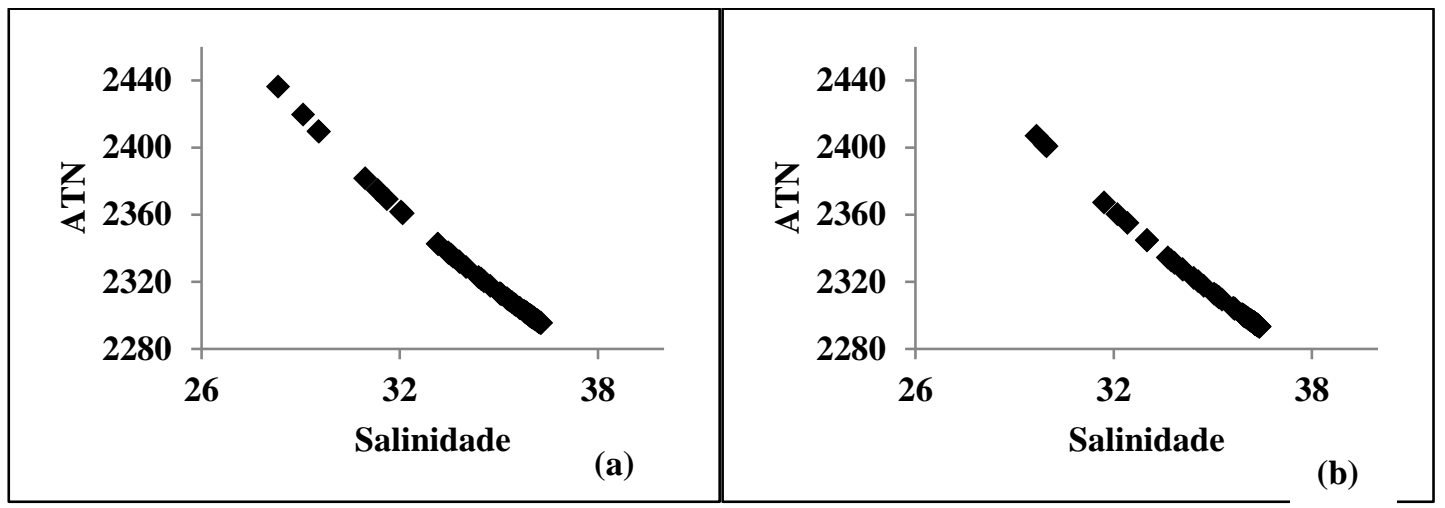

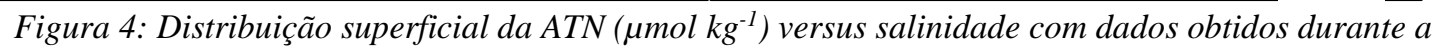
Operação Norte III (a) e Norte IV (b).

Este comportamento conservativo também foi observado para AT, com uma variação superficial de $1971 \mu$ mol kg $^{-1}$ a $2379 \mu$ mol kg$~^{-1}$ (média $2317 \mu \mathrm{mol} \mathrm{kg}^{-1} \pm$ 90,40), durante a Operação Norte III e de $1878 \mu \mathrm{mol} \mathrm{kg}^{-1}$ a $2386 \mu \mathrm{mol} \mathrm{kg}^{-1}$ (média de $2347 \mu \mathrm{mol} \mathrm{kg}^{-1} \pm 80,80$ ) durante a Operação Norte IV (Tabela 1).

A análise de componentes principais com os dados superficiais obtidos durante as Operações Norte III e Norte IV, mostrou forte relação entre a salinidade $(-0,97)$ e AT $(-0,98)$, e uma relação inversa deste grupo com a temperatura $(0,68)$ e a ATN $(0,97)$, ao longo da PC1, que explicou $34 \%$ da variância total (Tabela 3). Essa componente reforçou o verificado nas figuras $4 \mathrm{a}$ e $4 \mathrm{~b}$, ou seja, com o aumento da salinidade houve uma diminuição da ATN. Por outro lado, a relação inversa entre a temperatura e a salinidade a AT, indica a presença da massa de água costeira com baixa salinidade e alta temperatura.

Tabela 3: Pesos e variância explicada pelas duas primeiras componentes principais da análise dos parâmetros abióticos superficiais com dados obtidos durante as Operações Norte III e Norte IV. Os valores mais significativos estão em negrito.

\begin{tabular}{lcc}
\hline Variável & PC1 & PC2 \\
\hline Temperatura $\left({ }^{\circ} \mathrm{C}\right)$ & $\mathbf{0 , 6 8}$ & $-0,36$ \\
Salinidade & $\mathbf{- 0 , 9 7}$ & $-0,17$ \\
pH & 0,31 & $\mathbf{- 0 , 8 9}$ \\
AT $\left(\mu \mathrm{mol} \mathrm{kg}{ }^{-1}\right)$ & $\mathbf{- 0 , 9 8}$ & 0,17 \\
ATN $\left(\mu \mathrm{mol} \mathrm{kg}{ }^{-1}\right)$ & $\mathbf{0 , 9 7}$ & 0,20 \\
\hline Variância explicada $(\%)$ & 34 & 10 \\
\hline
\end{tabular}

Ao longo da PC2, que explicou $10 \%$ da variância total, destacou-se somente o pH $(-0,89)$. O aumento ou a diminuição deste parâmetro no sistema do carbonato é relacionado ao processo fotossintético e a oxidação da matéria orgânica (Tabela 3).

O valor superficial de $\mathrm{pH}$ variou de 7,48 a 8,34 (média 8,16 $\pm 0,17$ ) durante a Operação Norte III, e de 7,38 a 8,23 (média 7,75 $\pm 0,13$ ) durante a Operação Norte IV. Na coluna de água o mínimo foi de 7,10 e o máximo de 8,38 (média 8,05 \pm 0,24) durante a Operação Norte IIII, e de 7,10 e de 8,27 (média 7,70 $\pm 0,17$ ) durante a Operação Norte IV.

Com os dados obtidos na coluna de água e na superfície foi feita uma análise de componentes principais que explicou 39\% da variância total. Ao longo da PC1, que explicou $27 \%$ da variância total, mostrou uma relação direta entre a salinidade $(-0,98)$ e a AT $(-0,98)$, os quais foram inversamente relacionados com a ATN $(0,87)$. 
Ao longo da PC2 destacou-se a temperatura $(-0,76)$ e o $\mathrm{pH}(-0,79)$, esse componente explicou $12 \%$ da variância total, o que indica que com a diminuição da temperatura ocorreu um menor valor de $\mathrm{pH}$ (Tabela 4).

Tabela 4: Pesos e variância explicada pelas duas primeiras componentes principais da análise dos parâmetros abióticos (coluna de água e superfície) com dados obtidos durante as Operações Norte III e Norte IV. Os valores mais significativos estão em negrito.

\begin{tabular}{lcc}
\hline Variável & PC1 & PC2 \\
\hline Temperatura $\left({ }^{\circ} \mathrm{C}\right)$ & $-0,21$ & $\mathbf{- 0 , 7 6}$ \\
Salinidade & $\mathbf{- 0 , 9 8}$ & 0,03 \\
pH & $-0,01$ & $\mathbf{- 0 , 7 9}$ \\
AT $\left(\mu \mathrm{mol} \mathrm{kg}{ }^{-1}\right)$ & $\mathbf{- 0 , 9 8}$ & 0,03 \\
ATN $\left(\mu \mathrm{mol} \mathrm{kg}{ }^{-1}\right)$ & $\mathbf{0 , 8 7}$ & $-0,13$ \\
\hline Variância explicada $(\%)$ & 27 & 12 \\
\hline
\end{tabular}

\section{DISCUSSÃO}

$\mathrm{O}$ conhecimento da concentração da $\mathrm{AT}$ e do valor do $\mathrm{pH}$ do ambiente marinho é relacionado ao sistema do dióxido de carbono inorgânico (DCI). De acordo com Bakker et al. (1999) [17] o DCI na água do mar consiste aproximadamente de $0,5-1 \%$ de dióxido de carbono, de $0,002 \%$ de ácido carbônico, de $90 \%$ de bicarbonato e de $9 \%$ de carbonato.

Os bicarbonatos e carbonatos constituem aproximadamente $98 \%$ da $\mathrm{AT}$ em $\mathrm{pH}$ de 8,1 ; o que explica a relação linear entre a AT e a salinidade na camada superficial dos oceanos $[17,18,19$, 20]. Goyet et al. (1998) [21] descreveram que abaixo da camada superficial, a concentração de carbonato é afetada pela respiração, oxidação da matéria orgânica, e formação ou dissolução de carbonato de cálcio.

No presente trabalho os dados superficiais de AT ficaram dentro da faixa descrita por Millero et al. (1998) [19] para o Oceano Atlântico, valores entre $2180 \mu \mathrm{mol} \mathrm{kg}^{-1} \mathrm{e} 2459 \mu \mathrm{mol} \mathrm{kg}{ }^{-1}$. Contudo, os valores mínimos $\left(1971 \mu \mathrm{mol} \mathrm{kg} \mathrm{kg}^{-1} \mathrm{e} 1878 \mu \mathrm{mol} \mathrm{kg}{ }^{-1}\right.$, tabela 1$)$ detectados em ambas as operações foram menores do que os encontrados por Millero et al. (1998) [19], o que pode ser justificado pela presença de águas com baixos valores de salinidade (24, tabela 1) do que os considerados pelo referido autor (> 32). Goyet et al. (1998) [21] em pesquisa no Oceano Atlântico Tropical encontraram valores de AT entre $2260 \mu \mathrm{mol} . \mathrm{kg}^{-1}$ e $2450 \mu \mathrm{mol} . \mathrm{kg}^{-1}$, em águas com valores de salinidade entre 35 e 37 . Esses autores caracterizaram a Contracorrente Norte Equatorial com valores relativamente baixos de salinidade, e com baixas concentrações de $\mathrm{TCO}_{2}$ e AT; em contraste a Corrente Sul Equatorial foi caracterizada por altos valores de salinidade, de $\mathrm{TCO}_{2}$ e AT.

Os baixos valores de salinidade na área em estudo estão relacionados com presença da massa de Água Costeira consequência das águas oriundas do rio Amazonas. Essa massa de água teve valores elevados de temperatura e de ATN. Estes dois parâmetros foram positivamente correlacionados na análise de componentes principais obtida com os dados superficiais.

$\mathrm{Na}$ área oceânica, da superfície para o fundo, a primeira massa de água encontrada na região é a Água Tropical, com valores de ATN menores quando comparados aos da Água Costeira. Abaixo desta registra-se a presença da Água Central do Atlântico Sul, baixo valor de salinidade. No intervalo de $500 \mathrm{~m}$ a cerca de $1200 \mathrm{~m}$ de profundidade encontra-se a Água Intermediária da Antártica, com baixo valor de temperatura [16]. Ressalta-se que, estas massas de águas foram detectadas somente na operação Norte III.

A distribuição superficial da AT e da ATN em função da salinidade foram similares, com comportamento conservativo, corroborando com o descrito por Millero et al. (1998) [19]. A troca entre o oceano e atmosfera na camada superficial modifica o conteúdo de espécies de carbono inorgânico na água do mar, porém não altera a concentração da AT [17].

Na ZEE Norte, área da Plataforma Continental do Amazonas, o aumento do $\mathrm{pH}$ foi relacionado diretamente com o aumento da clorofila $a$ e do oxigênio dissolvido [22]. Esta distribuição foi 
similar à observada por DeMaster et al. (1996) [23] que encontraram nas águas superficiais da Plataforma Continental do Amazonas, um modelo coerente de distribuições superficiais de $\mathrm{pH}$, clorofila $a$, saturação de oxigênio dissolvido e o conteúdo de sílica biogênica, identificando uma área de alta produtividade, elevadas concentrações de oxigênio dissolvido, pH e sílica.

Valores alcalinos do $\mathrm{pH}$ foram observados tanto na superfície quanto na coluna de água, o que demonstrou o equilíbrio entre os íons ácidos e básicos, apesar da diminuição do $\mathrm{pH}$ para um valor mínimo de 7,10 (tabela 2), em ambas as operações. O pH nos oceanos diminui com o decréscimo da temperatura e com o aumento da pressão hidrostática. A formação de carbonatos e bicarbonatos (principalmente de cálcio) diminuem com o aumento da profundidade e da concentração de $\mathrm{CO}_{2}$ dissolvido na água, provocando um decréscimo no $\mathrm{pH}$ [24].

Conforme observado na análise de componentes principais os valores de $\mathrm{pH}$ diminuíram com a temperatura, demonstrando que a diminuição do valor de $\mathrm{pH}$ foi ocasionada pela hidrólise do $\mathrm{CO}_{2}$ produzida pela remineralização da matéria orgânica particulada em águas profundas.

\section{CONCLUSÃO}

Os dados demonstraram que o sistema do dióxido de carbono ao longo da ZEE Norte mantém o pH dentro da faixa esperada para o ambiente marinho. Os menores valores de $\mathrm{pH}$, de AT e de ATN na camada superficial, foram observados na área com influência da descarga do rio Amazonas, ou seja, com baixos valores de salinidade.

\section{AGRADECIMENTOS}

Ao Conselho Nacional de Desenvolvimento Científico e Tecnológico (CNPq). À tripulação do Navio Oceanográfico ANTARES (H40). Ao Ministério da Defesa (MD)/ Marinha do Brasil (MB), à Comissão Interministerial para os Recursos do Mar (CIRM), à Diretoria de Hidrografia e Navegação (DHN).

\section{REFERÊNCIAS BIBLIOGRÁFICAS}

1. Ramos CAS. Análises físico-químicas de sistemas marginais marinhos. Rio de Janeiro: Interciência; 2004. $128 \mathrm{p}$.

2. Pereira RC, Gomes SA. Biologia Marinha. Rio de Janeiro:Interciência; 2009. 656 p.

3. Burke CM, Atkinson MJ. Measurement of total alkalinity in hypersaline water: values of $\mathrm{f}_{\mathrm{H}}$. Marine Chemistry. 1988. 25:49-55, doi: 10.1016/0304-4203(88(90014-x.

4. Millero FJ. Chemical Oceanography. Florida: CRC Press; 2013. 591 p.

5. Wong GTF. Alkalinity and $\mathrm{pH}$ in the Southern Chesapeake Bay and the James River Estuary. Limnology and Oceanography. 1979. 24(5): 970-977, doi:10.4319/Io.1979.24.5.0970

6. Muller-Karger FE, Mcclain CR, Richardson PL. The dispersal of the Amazon's water. Nature. 1988. 333:56-69, doi: 10.1038/333056a0.

7. Kineke GC, Sternberg RW, Trowbridge JH, Geyer WR. Fluid-mud processes on the Amazon continental shelf. Continental Shelf Research. 1996. 16:667-696, doi:10.1016/0278-4343(95)00050-x.

8. Gibbs R.J. Water chemistry of the Amazon river. Geochim. Cosmochim. Acta. 1972. 36:1061-1066.

9. Curtin TB, Legeckis RV. Physical observations in the plume region of the Amazon River during peak discharge - I. Surface variability. Continental Shelf Research. 1986. 6 (1/2): 31-51, doi:10.106/02784343(86)90052-x.

10. Ryther JH, Menzel DW, Norwin N. Influence of the Amazon river outflow on the ecology of the western tropical atlantic I. Hydrography and nutrient chemistry. Journal of Marine Research. 1967. 25: 69- 83.

11. Santos MLS. Influência dos rios Amazonas e Pará sobre a biomassa fitoplanctônica [dissertação]. Recife (PE): Universidade Federal de Pernambuco; 2000. $108 \mathrm{f}$.

12. Santos MLS, Silva AC. Estudo da temperatura, salinidade e massas d'água na plataforma continental do Amazonas e área oceânica adjacente. In: Semana Nacional de Oceanografia; Itajaí (SC): Anais; 2000. p. 432- 434.

13. Delfino IB, Eschirque AS, Saraiva, ALL, Santos, MLS. Estudo dos parâmetros físicos e químicos na Plataforma Continental do Maranhão e área oceânica adjacente. In: Congresso Brasileiro de Geoquímica; Belém (PA): Anais;2003. 
14. Eschirque, AS, Saraiva, ALL, Delfino, IB, Santos, MLS, Silva AC. Sazonalidade na ZEE Norte: estudo de parâmetros abióticos ( $4^{\circ} 30^{\prime} \mathrm{N}$ e $6^{\circ} 46,2^{\prime} \mathrm{N}$ de latitude e $51^{\circ} 1,8^{\prime} \mathrm{W}$ e $49^{\circ} 21^{\prime} \mathrm{W}$ de longitude). In: Congresso Brasileiro de Geoquímica; Belém (PA): Anais;2003.

15. Wagener ALR, Carreira R.. Métodos Analíticos de Referência em Oceanografia Química - Programa REVIZEE. Rio de Janeiro: Ministério do Meio Ambiente; 1997. 83 p.

16. Silva AC, Araújo M, Bourlés B. Variacão Sazonal da Estrutura de Massa de água na Plataforma Continental do Amazonas e área Oceânicas Adjacentes. Revista Brasileira de Geofísica. 2005. 23(2): 145 157, doi: 10.1590/S0102-261X2005000200004.

17. Bakker DCE, Baar HJW, Jong E. The dependence on temperature and salinity of dissolved inorganic carbon in East Atlantic surface waters. Marine Chemistry. 1999. 65:263-280, doi:10.106/S03044206(99)00017-1.

18. Brewer PG, Bradshaw AL, Williams RT. Measurements of total carbon dioxide and alkalinity in the North Atlantic Ocean in 1981. In: Trabalka, J.R., Reichle, D.E. _Eds.., The changing carbon cycle. A global analysis, Springer-Verlag, 1986. pp. 348-370.

19. Millero FJ, Lee K, Roche M. Distribution of alkalinity in the surface waters of the major oceans. Marine Chemistry. 1998. 60:111-30, doi:10.106/S0304-4203(97)00084-4.

20. Goyet C, Brewer PG. Biochemical properties of the oceanic carbon cycle. In: Willebrand, J., Anderson, D.L.T. Eds.., NATO ASI Series, vol. I 11, Modeling Oceanic Climate Interactions, Springer-Verlag, Berlin, Heidelberg, 1993. p. 271-297.

21. Goyet C, Adams R, Eischeid G. Observations of the CO system properties in the tropical Atlantic Ocean. Marine Chemistry. 1998. 60:49-61.

22. Santos MLS, Muniz K, Barros Neto B, Feitosa FAN. Estudo das diferentes formas de fósforo nas águas da Plataforma Continental do Amazonas. Química Nova. 2007. 30: 569 - 573, doi:10.1590/S010040422007000300012.

23. DeMaster DJ, Smith WO, Aller JY. Biogeochemical processes in Amazon shelf waters: chemical distributions and uptake rate of silicon, carbon and nitrogen. Continental Shelf Research. 1996. 16(5/6): 617- 643, doi: 10.1016/0278-4343(95)00048-8.

24. Riley JP, Chester R. Introduction to marine chemistry. London: Academis Press; 1971. 465p. 